\title{
Fuzzy-Based Optimal Adaptive Line-of-Sight Path Following for Underactuated Unmanned Surface Vehicle with Uncertainties and Time-Varying Disturbances
}

\author{
Dongdong Mu $\mathbb{D}^{\mathbb{D}}$, Guofeng Wang $(\mathbb{D}$, Yunsheng Fan, Yiming Bai, and Yongsheng Zhao \\ School of Information Science and Technology, Dalian Maritime University, Dalian 116026, China \\ Correspondence should be addressed to Guofeng Wang; gfwangsh@163.com
}

Received 4 July 2017; Accepted 10 December 2017; Published 19 February 2018

Academic Editor: Haranath Kar

Copyright (C) 2018 Dongdong Mu et al. This is an open access article distributed under the Creative Commons Attribution License, which permits unrestricted use, distribution, and reproduction in any medium, provided the original work is properly cited.

\begin{abstract}
This paper investigates the path following control problem for an underactuated unmanned surface vehicle (USV) in the presence of dynamical uncertainties and time-varying external disturbances. Based on fuzzy optimization algorithm, an improved adaptive line-of-sight (ALOS) guidance law is proposed, which is suitable for straight-line and curve paths. On the basis of guidance information provided by LOS, a three-degree-of-freedom (DOF) dynamic model of an underactuated USV has been used to design a practical path following controller. The controller is designed by combining backstepping method, neural shunting model, neural network minimum parameter learning method, and Nussbaum function. Neural shunting model is used to solve the problem of "explosion of complexity," which is an inherent illness of backstepping algorithm. Meanwhile, a simpler neural network minimum parameter learning method than multilayer neural network is employed to identify the uncertainties and time-varying external disturbances. In particular, Nussbaum function is introduced into the controller design to solve the problem of unknown control gain coefficient. And much effort is made to obtain the stability for the closed-loop control system, using the Lyapunov stability theory. Simulation experiments demonstrate the effectiveness and reliability of the improved LOS guidance algorithm and the path following controller.
\end{abstract}

\section{Introduction}

USV is attracting more and more attention of researchers from all over the world because of its extensive applications in the military and civilian areas [1]. In military field, it can perform minesweeping, eavesdropping, tracking, and other missions in a harsh environment, where it is not suitable for people to participate in the tasks. It is worth mentioning that, in recent years, it often works with autonomous underwater vehicles (AUV) as a signal transfer platform [2-5]. In civilian field, with the rapid increase of population and the lack of land resources, people urgently need to acquire resources from seventy percent of the ocean surface area. This further intensifies the human determination to explore the ocean, so the development of unmanned surface vehicle is imminent. Trajectory tracking control enables the USV to track the desired time-referenced trajectory or virtual objects. For the path following problem, the control task is to follow a parameterized path without involving any requirements pertaining to temporal constraints. Taking into account the operating characteristics of USV and the actual situation in the ocean, it is more practical to study the path following control problem.

Generally speaking, the path following control for USV can be divided into three different system modules: navigation, guidance, and motion control [6], as shown in Figure 1. Navigation is with the function of directing a USV, for example, measuring its heading and speed. The implementation of these functions requires a variety of sensors and devices on the USV. Guidance is a module from a connecting link between the preceding and the following. It converts the desired path to desired heading, speed, and so on, to the motion control module. The design of motion controller is the last and most complicated step of path following. In the process of designing the controller, we need to consider many problems, such as model uncertainties, time-varying and 


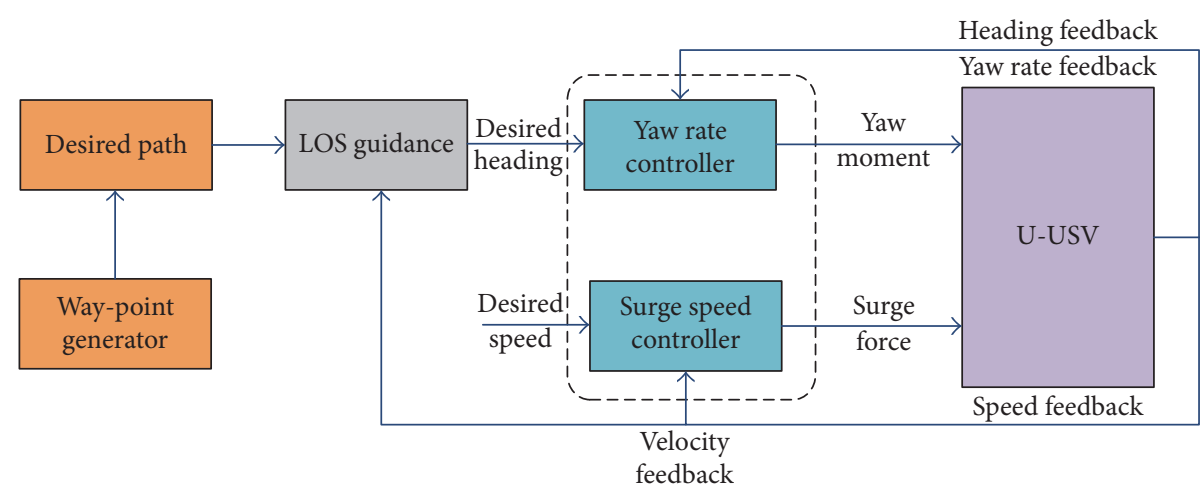

FIGURE 1: Visualization of the proposed path following strategy.

unmeasurable external disturbances, and unknown control gain. This paper focuses on the design and optimization of the latter two modules.

The LOS guidance law is the most widely used one in guidance module due to its simplicity and intuitiveness, and it had been applied to surface ships by McGookin et al. in [7]. However, in the presence of external disturbances caused by wind, waves, and ocean currents, the heading angle of USV is not equal to its course angle, and the traditional LOS algorithm can not deal with this problem. During path following, the difference between heading angle and course angle is sideslip angle. If sideslip angle is not compensated, it may enlarge the tracking errors and degrade the performance of the path following [8]. To solve the issue of sideslip angle and compensate for its influence, many efforts have been made by research institutions and individuals around the world. In [9], a fully actuated vessel was exposed to a constant environmental force, and a sideslip angle compensation was introduced to design path following controller. The guidance strategy and path following controller can be extended to underactuated vessels, but the paper did not mention how to obtain sideslip angle. In practical engineering, the simplest way is to directly measure the value of sideslip angle. In [10], the sideslip angle was computed by measuring sway and surge velocities. However, the corresponding sensor devices are often expensive, so scholars want to solve this problem through theoretical methods. Based on this, an integral term was added to the traditional LOS guidance algorithm proposing integral LOS (ILOS) to alleviate the effect of sideslip angle [11]. The work of [12] presented an extensive analysis of the ILOS guidance method for path following task of underactuated ships and showed that due to the embedded integral action the guidance law made the ships follow straight-lines by compensating for the sideslip angle effect of environmental disturbances. In [13], proportional LOS (PLOS) was first proved to be uniform local exponential stability (UGES) and uniform global asymptotic stability (UGAS), and it was used in a simplified vehicle model. In path following, we can consider the sideslip angle to be a constant or a slowly changing variable. In [14], ALOS was proposed to achieve the online compensation of sideslip angle. However, it considered only one error variable. Another drawback of traditional LOS is that its look-ahead distance $(\Delta)$ is a constant value. According to engineering experience, when approaching the target path, a larger $\Delta$ is needed, and when away from the target path, a smaller $\Delta$ is needed. In [15], a model predictive control (MPC) approach was used to optimize the value of $\Delta$. In [16], a constant $\Delta$ was replaced by a functional equation about the cross-track error. On the basis of [16], an improved equation about cross-track error was proposed [17].

From the point of view of the whole path following system, guidance module and motion control module are independent, so we can design the controller individually. In the field of motion control, there are many excellent algorithms used to design controllers [18-20]. In [21], Lyapunov's direct method and backstepping technology were combined to design a path following controller for an underactuated USV to follow a desired path at a desired speed. Point-topoint strategy and backstepping method were employed to design a nonlinear robust adaptive path following controller, and all error signals in the system were shown to be uniformly ultimately bounded (UUB) [22]. To cope with the so-called "explosion of complexity" problem of backstepping, dynamic surface control (DSC) technique was introduced into the path following controller design for USV [23, 24]. Meanwhile, neural shunting model can also be chosen to replace the role of DSC to solve above problem for the controller of USV [25]. In view of the unknown dynamics of the model, the most widely adopted approach is to use the universal approximation capability of neural network [26, 27] or fuzzy logic [28, 29]. In [30], the uncertainties of dynamics are compensated by the robust RBF neural network for a marine surface vessel. Nevertheless, the multilayer neural network represented by RBF undoubtedly increases the computational complexity of the controller.

Motivated by the above-mentioned observations, the goal of this article is that, based on ALOS algorithm with a time-varying $\Delta$, in the presence of external perturbations, model uncertainties, and unknown input gain, backstepping method, neural network minimum parameter learning method, neural shunting model, and Nussbaum function are used to design controller. The main contributions of this note can be summarized as follows:

(1) Fuzzy logic is hired to optimize the $\Delta$ of ALOS guidance law. 
(2) Neural shunting model and neural network minimum parameter learning method are used to deal with "explosion of complexity" and model uncertainties problems, respectively. The qualitative analysis of these two methods can reduce the computational burden of the controller to some extent.

(3) Nussbaum function is first applied to solve the problem of unknown gain of path following controller for USV.

The rest of the paper is organized as follows. The problem formulation and preliminaries are presented in Section 2. A fuzzy optimization of ALOS is proposed in Section 3. In Section 4, path following controller is designed. In Section 5, the stability of the whole set of control system is demonstrated. In Section 6, numerical simulations are implemented to demonstrate the correctness of the guidance and control strategies. Section 7 concludes this paper.

\section{Problem Formulation and Preliminaries}

2.1. Dynamic Model of Underactuated USV. According to past experience, the complex model increases the difficulty of controller design, and the simple model can not fully describe the physical characteristics of the object. So in this paper three-DOF dynamic model of underactuated USV is employed to describe its planar motion characteristics. The model of USV can be represented as [31]

$$
\begin{aligned}
\dot{x} & =u \cos \psi-v \sin \psi \\
\dot{y} & =u \sin \psi+v \cos \psi \\
\dot{\psi} & =r, \\
m_{11} \dot{u}-m_{22} v r+d_{11} u & =\tau_{u}+b_{u} \\
m_{22} \dot{v}+m_{11} u v+d_{22} v & =b_{v} \\
m_{33} \dot{r}-\left(m_{11}-m_{22}\right) u v+d_{33} r & =\tau_{r}+b_{r},
\end{aligned}
$$

where $(x, y)$ denotes the position of USV in the earth-fixed inertial frame, $\psi$ is heading angle, $u$ is the surge velocity, $v$ is the sway velocity, and $r$ is the yaw rate. $m_{11}, m_{22}, m_{33}, d_{11}, d_{22}$, and $d_{33}$ represent the corresponding hydrodynamic damping and inertia. $\tau_{u}$ and $\tau_{r}$ are the surge force and the yaw control moment, respectively. $b_{u}, b_{v}$, and $b_{r}$ are external disturbances caused by wind, waves, and currents in the corresponding directions.

Remark 1. In actual engineering, $\tau_{u}, \tau_{r}, b_{u}, b_{v}$, and $b_{r}$ are bounded. Besides, $m_{11}, m_{22}, m_{33}, d_{11}, d_{22}$, and $d_{33}$ are uncertain as a result of changes in operating conditions.

\subsection{Neural Network Minimum Parameter Learning Method.} $\mathrm{RBF}$ neural network is one of the most commonly used tools, which is used to approximate the unknown dynamics and uncertain parameters in the system. However, the computational complexity of multilayer neural network increases the difficulty in engineering implementation. In order to reduce the computational burden of the controller, on the basis of neural network, [32] proposed neural network minimum parameter learning method.

For any continuous function $f(x)$, when the RBF neural network approximation techniques are used, the $f(x)$ can be written as follows:

$$
f(x)=W^{T} h(x)+\varepsilon \quad \forall x \in \Omega_{x},
$$

where $\Omega_{x}$ is a compact set, $x$ is neural network input, $W$ is an ideal neural network weight, $h=\left[h_{1}, h_{2}, \ldots, h_{l}\right]$ is Gauss function output, and $\varepsilon$ is approximation error.

The essence of neural network minimum parameter learning method is that defining $\varphi=\|W\|^{2}$, and $\varphi$ is a normal number. $\widehat{\varphi}$ is the estimated value of $\varphi$, and its estimated error $\widetilde{\varphi}=\widehat{\varphi}-\varphi$. In this paper, $|\cdot|$ is the absolute operator of a scalar, and $\|\cdot\|$ is the Euclidean norm of a vector.

2.3. Neural Shunting Model. Neural shunting model belongs to the field of biology. In the early days, it was used to describe the responses of neurons to external stimuli. With the continuous progress of science and technology, it has been applied to path planning, mechanical arm control, and other fields $[33,34]$. In essence, neural shunting model is a filter that can flatten the system's signals.

The neural shunting model can be represented as

$$
\dot{\chi}=-A \chi+(B-\chi) f\left(\chi_{d}\right)-(D+\chi) g\left(\chi_{d}\right),
$$

where $\chi$ is output and $\chi_{d}$ is input. $A, B$, and $D$ are corresponding normal numbers. If $\chi_{d}>0, f\left(\chi_{d}\right)=\chi_{d}$ and $g\left(\chi_{d}\right)=0$; if $\chi_{d}<0, f\left(\chi_{d}\right)=0$ and $g\left(\chi_{d}\right)=-\chi_{d}$. In other words, $f\left(\chi_{d}\right)>0$ and $g\left(\chi_{d}\right)>0$.

2.4. Nussbaum Function. In order to deal with the unknown sign of the control gain, Nussbaum function [35] is introduced into the design of the controller.

If a continuous function $N(\bullet)$ satisfies the conditions

$$
\begin{aligned}
& \lim _{s \rightarrow \infty} \sup \frac{1}{s} \int_{0}^{s} N(\xi) d \xi=\infty \\
& \liminf _{s \rightarrow \infty} \frac{1}{s} \int_{0}^{s} N(\xi) d \xi=-\infty
\end{aligned}
$$

then $N(\bullet)$ is called a Nussbaum function, and the commonly used Nussbaum functions are $\xi^{2} \cos (\xi), \xi^{2} \sin (\xi)$, $\exp \left(\xi^{2}\right) \cos ((\pi / 2) \xi)$, and so on.

Lemma 2. Define $V(\bullet)$ and $\xi(\bullet)$ as smooth functions on $\left[0, t_{f}\right]$ and for $\forall t \in\left[0, t_{f}\right], V(t) \geq 0 . N(\bullet)$ is an even and smooth Nussbaum function, and $\theta_{0}$ is a nonzero constant. If the inequality

$$
V(t) \leq \int_{0}^{t}\left(\theta_{0} N(\xi)+1\right) \dot{\xi}(\mu) d \mu+C,
$$

where $\forall t \in\left[0, t_{f}\right)$ and $C$ is a constant, holds, then $V(t), N(\xi)$, and $\int_{0}^{t}\left(\theta_{0} N(\xi)+1\right) \dot{\xi}(\mu) d \mu$ must be bounded on $\left[0, t_{f}\right)$. For the proof of Lemma 2 refer to [36]. 


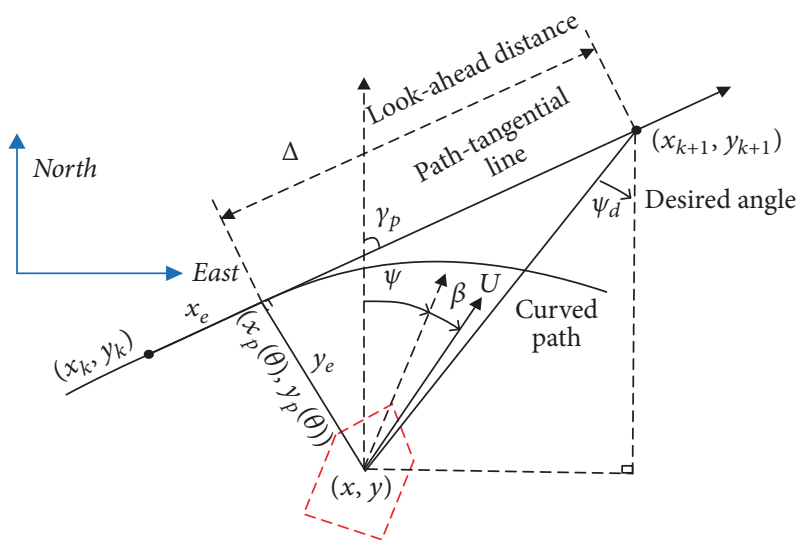

FIGURE 2: LOS guidance geometry for curved paths.

\section{LOS Guidance Algorithms}

3.1. ALOS. The LOS algorithm transforms the desired path into physical quantities that can be controlled, where the adaptive sideslip angle and the fuzzy optimization $\Delta$ are introduced in this chapter. In the field of ship motion control, the earth-fixed inertial frame and the body-fixed frame are used to describe USV motion states, such as position and direction. The schematic diagram of the LOS guidance is shown in Figure 2.

$(x, y)$ and $\left(x_{p}(\theta), y_{p}(\theta)\right)$ represent the actual location and target location of USV, respectively, where $\theta$ is an independent variable. The speed $U$ is manually or automatically controlled, which is assumed to be positive and bounded. $\psi$ represents the heading angle of USV. Besides, it is rotated at an angle $\gamma_{p}$.

$$
\gamma_{p}(\theta)=\operatorname{artan}\left(y_{p}^{\prime}(\theta), x_{p}^{\prime}(\theta)\right)
$$

where $x_{p}^{\prime}(\theta)=\partial x_{p} / \partial \theta$ and $y_{p}^{\prime}(\theta)=\partial y_{p} / \partial \theta$. It is worth noting that $\gamma_{p}$ is a constant value for a straight-line.

For the USV, the errors (along tracking error $x_{e}$ and cross-tracking error $\left.y_{e}\right)$ between $(x, y)$ and $\left(x_{p}(\theta), y_{p}(\theta)\right)$ are shown in

$$
\begin{aligned}
& x_{e}=\cos \left(\gamma_{p}\right)\left(x-x_{p}(\theta)\right)+\sin \left(\gamma_{p}\right)\left(y-y_{p}(\theta)\right) \\
& y_{e}=-\sin \left(\gamma_{p}\right)\left(x-x_{p}(\theta)\right)+\cos \left(\gamma_{p}\right)\left(y-y_{p}(\theta)\right)
\end{aligned}
$$

According to (1) and (2), the time derivative of (8) is

$$
\begin{aligned}
& \dot{x}_{e}=U \cos \left(\psi-\gamma_{p}+\beta\right)+\dot{\gamma}_{p} y_{e}-U_{p} \\
& \dot{y}_{e}=u \sin \left(\psi-\gamma_{p}\right)+v \cos \left(\psi-\gamma_{p}\right)+\dot{\gamma}_{p} x_{e}+\Xi,
\end{aligned}
$$

where $\beta=\operatorname{ar} \tan (v, u)$ represents the sideslip angle, $\Xi=$ $\dot{\theta} \sqrt{x^{\prime}(\theta)^{2}+y^{\prime}(\theta)^{2}} \sin \left(\gamma_{p}+\phi\right)$, and $\phi=a \tan 2\left(-y^{\prime}(\theta)\right.$, $\left.x^{\prime}(\theta)\right)=-\gamma_{p}$. Meanwhile, $U_{p}$ is considered as the virtual reference point to stabilize $x_{e}$.

Assumption 3. If $\beta$ is small and slow time-varying, $\dot{\beta}=0$, $\sin (\beta)=\beta$, and $\cos (\beta)=1 . \widehat{\beta}$ is the estimated value of $\beta$ and $\widetilde{\beta}=\beta-\widehat{\beta}$ is the estimation error.
The ALOS guidance algorithm can be chosen as

$$
\begin{gathered}
\psi_{d}=\gamma_{p}-\operatorname{acr} \tan \left(\frac{1}{\Delta} y_{e}+\widehat{\beta}\right) \\
\dot{\hat{\beta}}=\kappa \frac{U y_{e}}{\sqrt{\Delta^{2}+\left(y_{e}+\Delta \widehat{\beta}\right)^{2}}},
\end{gathered}
$$

where $\kappa$ is a constant greater than zero. In order to stabilize $x_{e}, U_{p}$ can be chosen as

$$
\begin{aligned}
& U_{p}=-U \frac{\Delta}{\sqrt{\Delta^{2}+\left(y_{e}+\Delta \widehat{\beta}\right)^{2}}}+\gamma x_{e} \\
& \dot{\theta}=\frac{U_{p}}{\sqrt{x_{p}^{\prime}(\theta)^{2}+y_{p}^{\prime}(\theta)^{2}}},
\end{aligned}
$$

where $0<\Delta_{\min }<\Delta<\Delta_{\max }$ is the user specified look-ahead distance and $\gamma$ is a constant greater than zero.

Proof. The origin $\left(x_{e}, y_{e}, \widetilde{\beta}\right)=(0,0,0)$ is UGES if $\theta$ is undated by (13), $\psi_{d}$ is equal to (10), and $U_{p}$ satisfies (12).

This is because $\sin \left(\arctan \left(-\left(y_{e}+\Delta \widehat{\beta}\right) / \Delta\right)\right)=-\left(y_{e}+\right.$ $\Delta \widehat{\beta}) / \sqrt{\Delta^{2}+\left(y_{e}+\Delta \widehat{\beta}\right)^{2}}$ and $\cos \left(\arctan \left(-\left(y_{e}+\Delta \widehat{\beta}\right) / \Delta\right)\right)=$ $-\Delta / \sqrt{\Delta^{2}+\left(y_{e}+\Delta \widehat{\beta}\right)^{2}}$. One can get that

$$
\begin{aligned}
& \dot{x}_{e}=\dot{\gamma}_{p} y_{e}-U \frac{\Delta}{\sqrt{\Delta^{2}+\left(y_{e}+\Delta \widehat{\beta}\right)^{2}}}-U_{p} \\
& \dot{y}_{e}=-U \frac{y_{e}-\Delta \tilde{\beta}}{\sqrt{\Delta^{2}+\left(y_{e}+\Delta \widehat{\beta}\right)^{2}}}-\dot{\gamma}_{p} x_{e} .
\end{aligned}
$$

Design the Lyapunov function $V_{1}$.

$$
V_{1}=\frac{1}{2} x_{e}^{2}+\frac{1}{2} y_{e}^{2}+\frac{1}{2 \kappa} \widetilde{\beta}^{2}
$$

Using (11) and (14), the time derivative of (15) is

$$
\dot{V}_{1}<-\gamma x_{e}^{2}-\frac{U y_{e}^{2}}{\sqrt{\Delta^{2}+\left(y_{e}+\Delta \widehat{\beta}\right)^{2}}}<0 .
$$

We can choose that $U=l \sqrt{\Delta^{2}+\left(y_{e}+\Delta \widehat{\beta}\right)^{2}}$, where $l$ is a constant greater than zero. Then one can get that

$$
\dot{V}_{1} \leq-\gamma x_{e}^{2}-l y_{e}^{2}<0 .
$$

$V_{1}$ is positive define and radially unbounded, while its derivative is quadratically negative. Thus, we can follow the definition of Lyapunov arguments and know that the guidance system at origin $\left(x_{e}, y_{e}, \widetilde{\beta}\right)=(0,0,0)$ is UGES. 


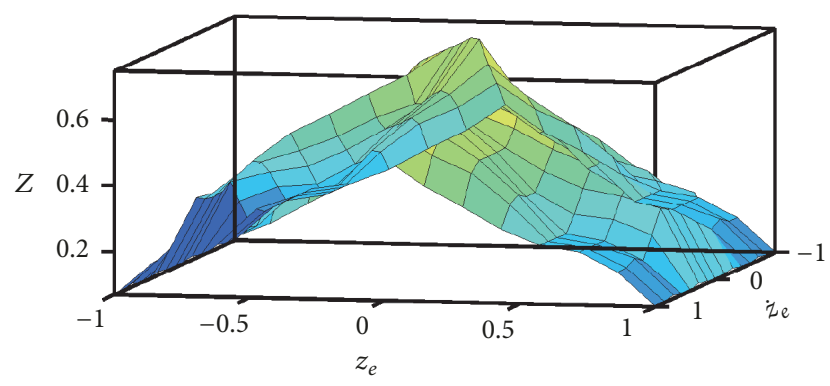

FIGURE 3: Fuzzy control rules.

3.2. Fuzzy Optimization Look-Ahead Distance. In this subchapter, fuzzy logic is used to optimize the value of $\Delta$. The principle of optimizing $\Delta$ is that when USV is farther away from the desired path, a smaller $\Delta$ is selected; on the contrary, a larger $\Delta$ is selected when USV is near the desired path. $z_{e}=\sqrt{x_{e}^{2}+y_{e}^{2}}$ and $\dot{z}_{e}$ are used as inputs to fuzzy logic. The gain $N$ is output. Then $\Delta=\Delta_{\min }+N *\left(\Delta_{\max }-\Delta_{\min }\right)$.

(1) $z_{e}$ and $\dot{z}_{e}$ are normalized to $\left[\begin{array}{ll}-1 & 1\end{array}\right]$, and the data domain of $N$ is [ 0101$]$.

(2) The fuzzy subsets of $z_{e}, \dot{z}_{e}$, and $N$ are [NB, NS, Z, PS, $\mathrm{PB}]$.

(3) The control rules are shown in Figure 3.

(4) Fuzzy reasoning adopts Zadeh and max-min, and defuzzification uses the method of centroid area center of gravity.

\section{Controller Design}

Yaw rate controller and surge speed controller are designed to complete USV path following when the guidance module has been designed. Controller design is the most difficult part of the whole path following system. Many practical conditions need to be taken into account.

Assumption 4. In the control system, the first- and secondorder derivatives of all the error variables and reference signals are bounded.

Assumption 5. All variables needed (such as position, speed, and direction) of the USV are available for feedback.

4.1. Yaw Rate Controller. The goal of designing yaw rate controller is to make the actual heading follow the target value well. In other words, the heading error $\psi_{e}$ needs to be near zero.

Define

$$
\psi_{e}=\psi-\psi_{d}
$$

By differentiating $\psi_{e}$ in (18), we can get that

$$
\dot{\psi}_{e}=r-\dot{\psi}_{d} .
$$

For $\psi_{e} \rightarrow 0, \alpha_{r}$ is selected as virtual control.

$$
\alpha_{r}=-k_{1} \psi_{e}+\dot{\psi}_{d}
$$

where $k_{1}$ is a parameter greater than zero. Make $\alpha_{r}$ pass through neural shunting model (4) and define

$$
r_{e}=r-\chi
$$

Using (2) and (4), the time derivative of (21) is

$$
\dot{r}_{e}=f_{r}+\frac{1}{m_{33}} \tau_{r}+\frac{1}{m_{33}} b_{r}+\Theta,
$$

where $f_{r}=\left(\left(m_{11}-m_{22}\right) / m_{33}\right) u v-\left(d_{33} / m_{33}\right) r$ and $\Theta=([A+$ $\left.\left.f\left(\alpha_{r}\right)+g\left(\alpha_{r}\right)\right] \chi-\left[B f\left(\alpha_{r}\right)-D g\left(\alpha_{r}\right)\right]\right)$.

$f_{r}+\left(1 / m_{33}\right) b_{r}$ is approached by neural network minimum parameter learning method and its approximation error is $\varepsilon_{r}$ and $\left\|\varepsilon_{r}\right\| \leq \bar{\varepsilon}_{r}, \bar{\varepsilon}_{r}>0$.

The corresponding control law is chosen as

$$
\begin{aligned}
& \tau_{r}=N\left(\xi_{r}\right)\left(k_{r e} r_{e}+\frac{1}{2} r_{e} \widehat{\varphi}_{r} h^{T} h+\Theta+\psi_{e}\right) \\
& \dot{\xi}_{r}=r_{e}\left(k_{r e} r_{e}+\frac{1}{2} r_{e} \widehat{\varphi}_{r} h^{T} h+\Theta+\psi_{e}\right),
\end{aligned}
$$

where $k_{r e}$ is a parameter greater than zero, $N\left(\xi_{r}\right)$ is a Nussbaum function, and $h$ is shorthand for $h(x)$.

The adaptive law of neural network minimum parameter learning method is

$$
\dot{\hat{\varphi}}_{r}=\frac{1}{2} \gamma_{r} r_{e}^{2} h^{T} h-\kappa_{r} \gamma_{r} \widehat{\varphi}_{r}
$$

where $\gamma_{r}$ and $\kappa_{r}$ are arguments greater than zero.

\subsection{Surge Speed Controller}

Assumption 6. In this article, one assumes that $|v|$ is just a small amount compared to $U$, and this assumption is also very reasonable in practical engineering.

When time $t \rightarrow \infty$, the objective of then speed is $\lim _{t \rightarrow \infty} U \rightarrow U_{d}$. However, according to Assumption 6, the objective of the surge speed controller becomes $\lim _{t \rightarrow \infty} u \rightarrow$ $u_{d}$. Define a surge speed tracking error $u_{e}$ as

$$
u_{e}=u-u_{d}
$$

Using (2), the time derivative of (26) is

$$
\dot{u}_{e}=f_{u}+\frac{1}{m_{11}} \tau_{u}+\frac{1}{m_{11}} b_{u}-\dot{u}_{d}
$$

where $f_{u}=\left(m_{22} / m_{11}\right) v r-\left(d_{11} / m_{11}\right) u$.

$f_{u}+\left(1 / m_{11}\right) b_{u}$ is approached by neural network minimum parameter learning method and its approximation error is $\varepsilon_{u}$ and $\left\|\varepsilon_{u}\right\| \leq \bar{\varepsilon}_{u}, \bar{\varepsilon}_{u}>0$.

The corresponding control law is chosen as

$$
\begin{aligned}
& \tau_{u}=N\left(\xi_{u}\right)\left(k_{u e} u_{e}+\frac{1}{2} u_{e} \widehat{\varphi}_{u} h^{T} h-\dot{u}_{d}\right) \\
& \dot{\xi}_{u}=u_{e}\left(k_{u e} u_{e}+\frac{1}{2} u_{e} \widehat{\varphi}_{u} h^{T} h-\dot{u}_{d}\right),
\end{aligned}
$$


where $k_{u e}$ is a parameter greater than zero, $N\left(\xi_{u}\right)$ is a Nussbaum function, and $h$ is shorthand for $h(x)$.

The adaptive law of neural network minimum parameter learning method is

$$
\dot{\hat{\varphi}}_{u}=\frac{1}{2} \gamma_{u} u_{e}^{2} h^{T} h-\kappa_{u} \gamma_{u} \widehat{\varphi}_{u}
$$

where $\gamma_{u}$ and $\kappa_{u}$ are arguments greater than zero.

Remark 7. In previous literature, there are several ways to deal with control gain: (1) the parameter adaptive method is used to regulate control gain; (2) the neural network or fuzzy adaptive method is used to estimate control gain, but the problem is that these two methods difficultly avoid the singular problem; (3) no operation is done for the control gain. In this article, Nussbaum function is used to solve the problem of unknown control gain, and it can avoid the singular problem caused by the adaptive method.

Remark 8. This paper mainly reduces computation from three aspects: (1) traditional multilayer neural network is replaced by neural network minimum parameter learning method; (2) neural shunting model is used to solve the problem of "explosion of complexity"; (3) external disturbances and model uncertainties are considered as a whole, compensated by neural network minimum parameter learning method together.

\section{Lyapunov-Based Stability Analysis}

The stability of proposed control strategy and the closed-loop path following system are demonstrated in this chapter.

5.1. Stability of Proposed Control Strategy. Define error variable

$$
y_{r}=\chi-\alpha_{r}
$$

The time derivative of $y_{r}$ is obtained in (32) along (4).

$$
\dot{y}_{r}=-A_{r} \chi+B \alpha_{r}-X_{1} \text {, }
$$

where $A_{r}=A+f\left(\alpha_{r}\right)+g\left(\alpha_{r}\right)$ and $X_{1}=\left(\partial \alpha_{r} / \partial e_{1}\right) \dot{e}_{1}+$ $\left(\partial \alpha_{r} / \partial \dot{\psi}_{d}\right) \ddot{\psi}_{d},\left|X_{1}\right| \leq \bar{X}_{1}$ and $\bar{X}_{1}>0$.

Theorem 9. Consider the kinematic and dynamic models of underactuated USV which are given by (1) and (2). The proposed path following control laws (23) and (28) and adaptive laws (25) and (30) can guarantee that all errors are semiglobally uniformly ultimately bounded.

Proof. Define the second Lyapunov function candidate.

$$
V_{2}=\frac{1}{2}\left(\psi_{e}^{2}+u_{e}^{2}+r_{e}^{2}+y_{r}^{2}+\frac{1}{\gamma_{u}} \tilde{\varphi}_{u}^{2}+\frac{1}{\gamma_{r}} \tilde{\varphi}_{r}^{2}\right) .
$$

The time derivative of (33) is

$$
\dot{V}_{2}=\psi_{e} \dot{\psi}_{e}+u_{e} \dot{u}_{e}+r_{e} \dot{r}_{e}+y_{r} \dot{y}_{r}+\frac{1}{\gamma_{u}} \widetilde{\varphi}_{u} \dot{\hat{\varphi}}_{u}+\frac{1}{\gamma_{r}} \widetilde{\varphi}_{r} \dot{\hat{\varphi}}_{r} .
$$

Substituting (23) and (28) into (34) yields

$$
\begin{aligned}
\dot{V}_{2} \leq & -\left(k_{1}-1\right) \psi_{e}^{2}+\frac{1}{4} y_{r}^{2} \\
& +u_{e}\left(W_{u}^{T} h+\varepsilon_{u}+\frac{1}{m_{11}} \tau_{u}-\dot{u}_{d}\right) \\
& +r_{e}\left(W_{r}^{T} h+\varepsilon_{r}+\frac{1}{m_{33}} \tau_{r}+\Theta+\psi_{e}\right)+y_{r} \dot{y}_{r} \\
& +\frac{1}{\gamma_{u}} \tilde{\varphi}_{u} \dot{\hat{\varphi}}_{u}+\frac{1}{\gamma_{r}} \widetilde{\varphi}_{r} \dot{\hat{\varphi}}_{r} .
\end{aligned}
$$

Because $\left(\widetilde{\varphi}_{l}+\varphi_{l}\right)^{2} \geq 0, \widetilde{\varphi}_{l}^{2}+\varphi_{l}^{2}+2 \widetilde{\varphi}_{l}\left(\widehat{\varphi}_{l}-\widetilde{\varphi}_{l}\right) \geq 0$, we can get that $2 \widetilde{\varphi}_{\iota} \widehat{\varphi}_{\iota} \geq \widetilde{\varphi}_{\iota}^{2}-\varphi_{\iota}^{2}$, where $\iota=u$ and $r$. Then

$$
\begin{aligned}
\dot{V}_{2} \leq & -\left(k_{1}-1\right) \psi_{e}^{2}-\left(k_{u e}-\frac{1}{2}\right) u_{e}^{2}-\left(k_{r e}-\frac{1}{2}\right) r_{e}^{2} \\
& -\frac{1}{2} \kappa_{u} \widetilde{\varphi}_{u}^{2}-\frac{1}{2} \kappa_{r} \widetilde{\varphi}_{r}^{2}+\left(\frac{1}{m_{11}} N\left(\xi_{u}\right)+1\right) \dot{\xi}_{u} \\
& +\left(\frac{1}{m_{33}} N\left(\xi_{r}\right)+1\right) \dot{\xi}_{r}+y_{r} \dot{y}_{r}+\frac{1}{2} \kappa_{u} \varphi_{u}^{2} \\
& +\frac{1}{2} \kappa_{r} \varphi_{r}^{2}+1+\frac{1}{4} y_{r}^{2}+\frac{1}{2} \bar{\varepsilon}_{u}^{2}+\frac{1}{2} \bar{\varepsilon}_{r}^{2} .
\end{aligned}
$$
that

Define $B=A_{r}$; then $A_{r} \chi-A_{r} \alpha_{r}=A_{r} y_{r}$. One can get

$$
\begin{aligned}
\dot{V}_{2} \leq & -\left(k_{1}-1\right) \psi_{e}^{2}-\left(k_{u e}-\frac{1}{2}\right) u_{e}^{2}-\left(k_{r e}-\frac{1}{2}\right) r_{e}^{2} \\
& -\frac{1}{2} \kappa_{u} \widetilde{\varphi}_{u}^{2}-\frac{1}{2} \kappa_{r} \widetilde{\varphi}_{r}^{2}+\left(\frac{1}{m_{11}} N\left(\xi_{u}\right)+1\right) \dot{\xi}_{u} \\
& +\left(\frac{1}{m_{33}} N\left(\xi_{r}\right)+1\right) \dot{\xi}_{r}-A_{r} y_{r}^{2}-y_{r} X_{1}+\frac{1}{2} \kappa_{u} \varphi_{u}^{2} \\
& +\frac{1}{2} \kappa_{r} \varphi_{r}^{2}+1+\frac{1}{4} y_{r}^{2}+\frac{1}{2} \bar{\varepsilon}_{u}^{2}+\frac{1}{2} \bar{\varepsilon}_{r}^{2} .
\end{aligned}
$$

From Young's inequality, we have $a b \leq(1 / 2 \sigma) a^{2}+(\sigma / 2) b^{2}$ with $\sigma>0$ and $(a, b) \in \mathfrak{R}^{2}$; it follows that

$$
-y_{r} X_{1} \leq \frac{\sigma y_{r}^{2}}{2}+\frac{\bar{X}_{1}^{2}}{2 \sigma}
$$

Then

$$
\begin{aligned}
\dot{V}_{2} \leq & -\left(k_{1}-1\right) \psi_{e}^{2}-\left(k_{u e}-\frac{1}{2}\right) u_{e}^{2}-\left(k_{r e}-\frac{1}{2}\right) r_{e}^{2} \\
& -\frac{1}{2} \kappa_{u} \widetilde{\varphi}_{u}^{2}-\frac{1}{2} \kappa_{r} \widetilde{\varphi}_{r}^{2}-\left(A_{r}-\frac{\sigma}{2}-\frac{1}{4}\right) y_{r}^{2} \\
& +\left(\frac{1}{m_{11}} N\left(\xi_{u}\right)+1\right) \dot{\xi}_{u}+\left(\frac{1}{m_{33}} N\left(\xi_{r}\right)+1\right) \dot{\xi}_{r} \\
& +\frac{X_{1}^{2}}{2 \sigma}+\frac{1}{2} \kappa_{u} \varphi_{u}^{2}+\frac{1}{2} \kappa_{r} \varphi_{r}^{2}+\frac{1}{2} \bar{\varepsilon}_{u}^{2}+\frac{1}{2} \bar{\varepsilon}_{r}^{2}+1 .
\end{aligned}
$$


Define $\lambda_{1}=k_{1}-1>0, \lambda_{2}=k_{u e}-1 / 2>0, \lambda_{3}=k_{r e}-1 / 2>$ $0, \lambda_{4}=(1 / 2) \kappa_{u}, \lambda_{5}=(1 / 2) \kappa_{r}, \lambda_{6}=A_{r}-\sigma / 2-1 / 4>0$, $\Pi=\left(\left(1 / m_{11}\right) N\left(\xi_{u}\right)+1\right) \dot{\xi}_{u}+\left(\left(1 / m_{33}\right) N\left(\xi_{r}\right)+1\right) \dot{\xi}_{r}, \Gamma=\bar{X}_{1}^{2} / 2 \sigma+$ $(1 / 2) \bar{\varepsilon}_{u}^{2}+(1 / 2) \bar{\varepsilon}_{r}^{2}+(1 / 2) \kappa_{u} \varphi_{u}^{2}+(1 / 2) \kappa_{r} \varphi_{r}^{2}+1$.

From (39), we can get

$$
\begin{aligned}
\dot{V}_{2} \leq & -\lambda_{1} \psi_{e}^{2}-\lambda_{2} u_{e}^{2}-\lambda_{3} r_{e}^{2}-\lambda_{4} \widetilde{\varphi}_{u}^{2}-\lambda_{5} \widetilde{\varphi}_{r}^{2}-\lambda_{6} y_{r}^{2} \\
& +\Pi+\Gamma .
\end{aligned}
$$

Define $\lambda=\min \left\{\lambda_{1}, \lambda_{2}, \lambda_{3}, \lambda_{4}, \lambda_{5}, \lambda_{6}\right\}$; then if follows from (40) that

$$
\dot{V}_{2} \leq-2 \lambda V_{2}+\int_{0}^{t} \Pi d \mu+C+\Gamma .
$$

Solving inequality (41) gives

$$
V_{2} \leq V_{2}(0) e^{-\frac{2}{t}}+\frac{\int_{0}^{t} \Pi d \mu+C+\Gamma}{2 \lambda}, \quad \forall t>0 .
$$

Then the above inequality means that $V_{2}$ is eventually bounded by $\left(\int_{0}^{t} \Pi d \mu+C+\Gamma\right) / 2 \lambda$. It is known by Lemma 2 that, in the $\left[0, t_{f}\right], V_{2}(t), \xi(t)$, and $\int_{0}^{t} \Pi d \mu$ are bounded. Then $\psi_{e}, u_{e}, r_{e}, \widetilde{\varphi}_{u}, \widetilde{\varphi}_{r}, y_{r}$ are all bounded. In other words, there is no escape phenomenon. According to the Barbalat lemma [37], we can get that $\lim _{t \rightarrow \infty} \psi_{e}(t)=\lim _{t \rightarrow \infty} u_{e}(t)=\lim _{t \rightarrow \infty} r_{e}(t)=$ 0 . Eventually, the actual path of the USV can follow the desired path asymptotically.

\subsection{Stability of Closed-Loop Path Following System}

Proof. To analyze the stability of closed-loop system, construct the following Lyapunov function.

$$
V=V_{1}+V_{2}
$$

Its time derivative is computed as

$$
\begin{aligned}
\dot{V} \leq & -\gamma x_{e}^{2}-l y_{e}^{2}-\left(k_{1}-1\right) \psi_{e}^{2}-\left(k_{u e}-\frac{1}{2}\right) u_{e}^{2} \\
& -\left(k_{r e}-\frac{1}{2}\right) r_{e}^{2}-\frac{1}{2} \kappa_{u} \widetilde{\varphi}_{u}^{2}-\frac{1}{2} \kappa_{r} \widetilde{\varphi}_{r}^{2} \\
& -\left(A_{r}-\frac{\sigma}{2}-\frac{1}{4}\right) y_{r}^{2}-\left(K_{e u}-\frac{1}{2}\right) e_{u}^{2} \\
& -\left(K_{e r}-\frac{1}{2}\right) e_{r}^{2}+\nabla,
\end{aligned}
$$

where $\nabla=\left(\left(1 / m_{11}\right) N\left(\xi_{u}\right)+1\right) \dot{\xi}_{u}+\left(\left(1 / m_{33}\right) N\left(\xi_{r}\right)+1\right) \dot{\xi}_{r}+$ $\bar{X}_{1}^{2} / 2 \sigma+1+(1 / 2) \bar{\varepsilon}_{u}^{2}+(1 / 2) \bar{\varepsilon}_{r}^{2}+(1 / 2) \kappa_{u} \varphi_{u}^{2}+(1 / 2) \kappa_{r} \varphi_{r}^{2}$.

Therefore, all signals in the closed-loop network are UUB.

Remark 10. Nussbaum function is widely used in dealing with the problem of unknown control gain $[38,39]$. In [40], Nussbaum function was first applied to solve the problem of unknown control gain. Since then, it has gradually been

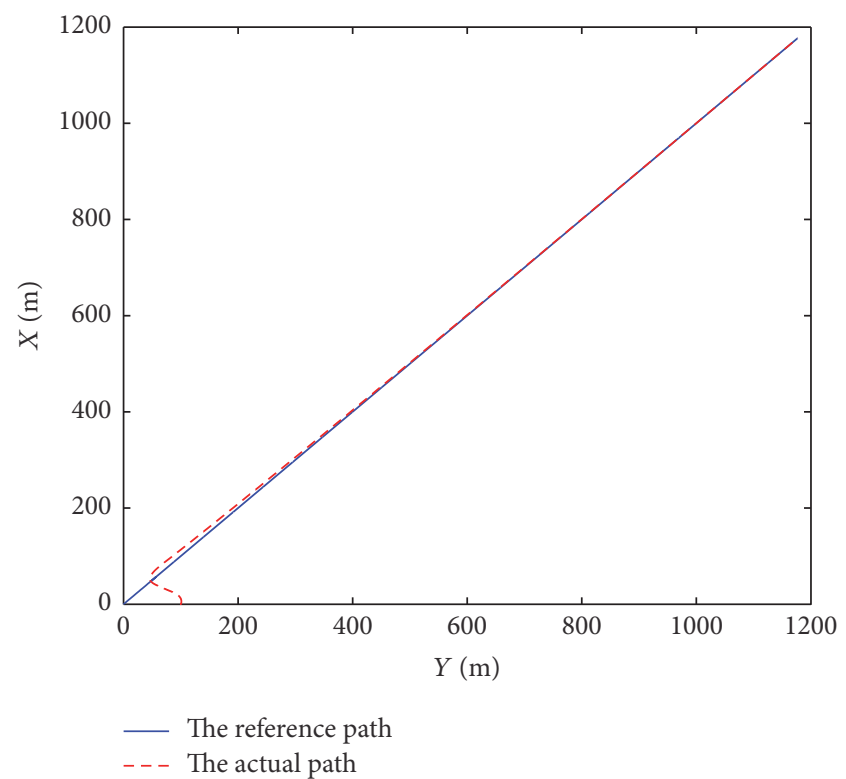

FIGURE 4: Path following performance (straight-line).

introduced into the controller design of engineering objects. In [41], Nussbaum function was introduced to design missile controller to solve the problem of uncertain coefficient. Meanwhile, the control of nonlinear systems often requires the application of Nussbaum function [42]. It is worth mentioning that Nussbaum function was first applied to ship's heading control by Professor Du and Guo [43]. The author of this article extends it to the USV's path following control. The proof of Nussbaum function's stability is shown in Section 2.4. For more detailed proof of the process, please refer to $[36,40]$.

\section{Numerical Simulations}

In this section, numerical simulations on CyberShip II are carried out to prove the correctness and effectiveness of the whole path following system. CyberShip II is a 1:70 scale replica of a supply ship, and for its main parameters please refer to [44]. Straight-line path following and curve path following are simulated, respectively.

6.1. Straight-Line Path Following Simulation. First, a straightline path following simulation is carried out and its desired geometrical path is expressed as $p_{d}(\theta)=[\theta, \theta]^{T}$. The control parameters are selected as follows: $u_{d}=1 \mathrm{~m} / \mathrm{s}, \kappa=0.01$, $\gamma=80, \Delta_{\min }=4, \Delta_{\max }=7, k_{1}=30, k_{u e}=500, k_{r e}=600$, $\gamma_{u}=800, \kappa_{u}=0.1, \gamma_{r}=800, \kappa_{r}=0.1, A=B=D=3$. The initial state of USV is $[x(0), y(0), \psi(0), u(0), v(0), r(0)]=$ $[0,100,1,0,0,0]$. According to [45], time-varying external disturbances are considered as

$$
\begin{aligned}
& b_{u}=1+0.1 \sin (0.2 t)+0.3 \cos (0.5 t) . \\
& b_{v}=1+0.2 \sin (0.2 t)+0.1 \cos (0.4 t) \\
& b_{r}=1+0.1 \sin (0.1 t)+0.1 \cos (0.2 t)
\end{aligned}
$$

Straight-line path simulation results are plotted in Figures 4-10. 


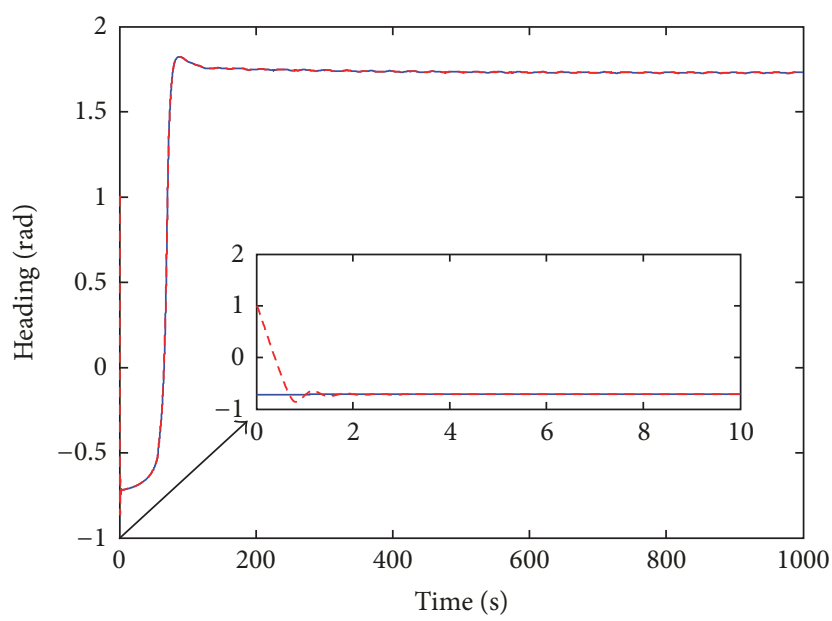

— The reference heading

-. - The actual heading

FIGURE 5: Heading control performance (straight-line).

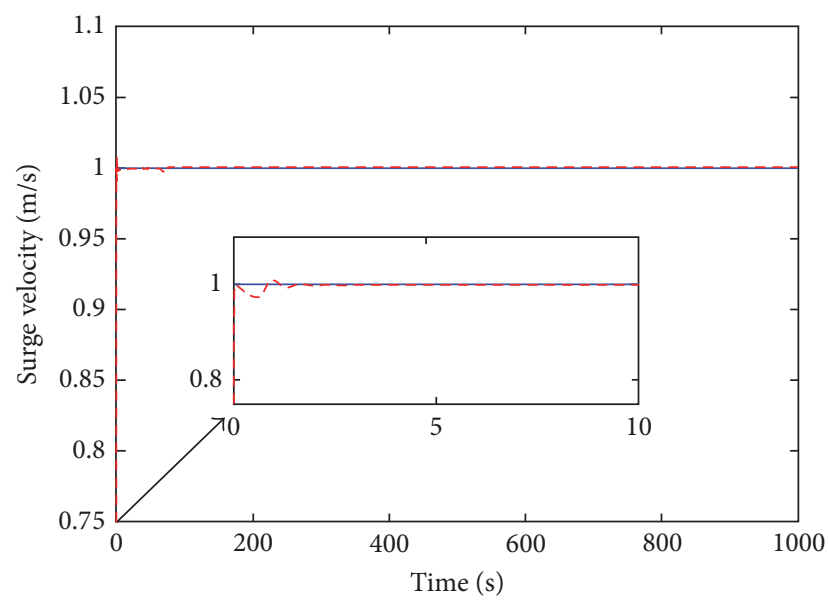

- The reference velocity

- - - The actual velocity

FIGURE 6: Speed control performance (straight-line).

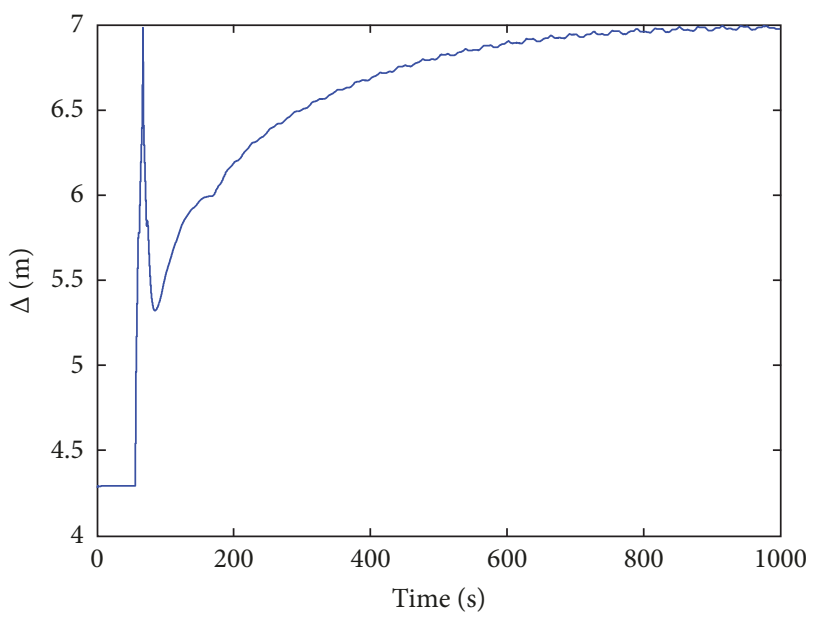

FIgURE 7: The value of $\Delta$ (straight-line).

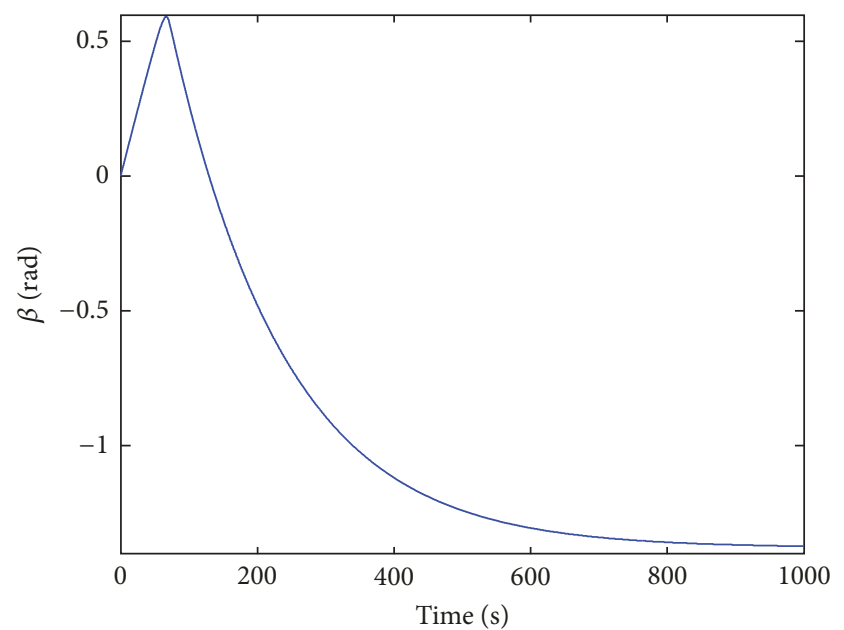

FIGURE 8: The estimated value of sideslip angle (straight-line).

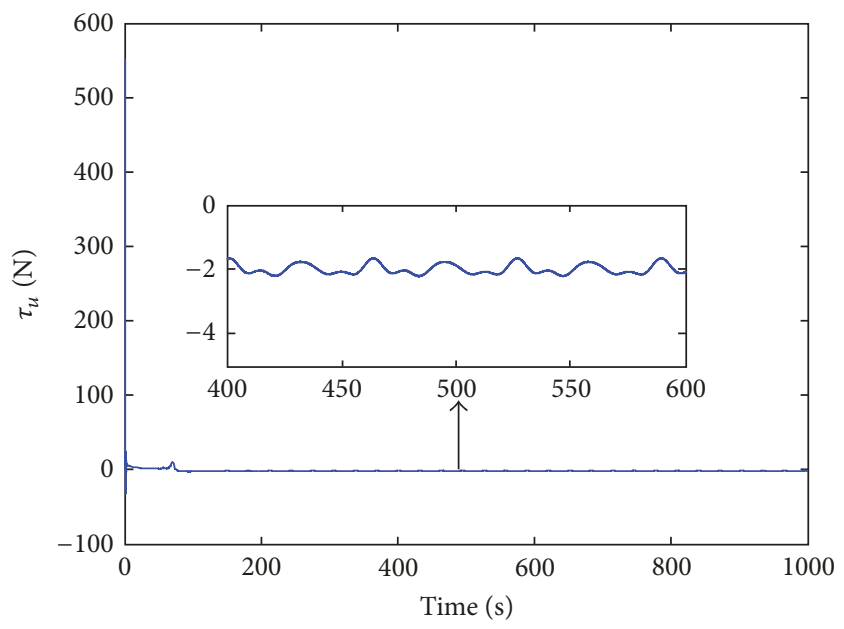

FIGURE 9: Control input $\tau_{u}$ (straight-line).

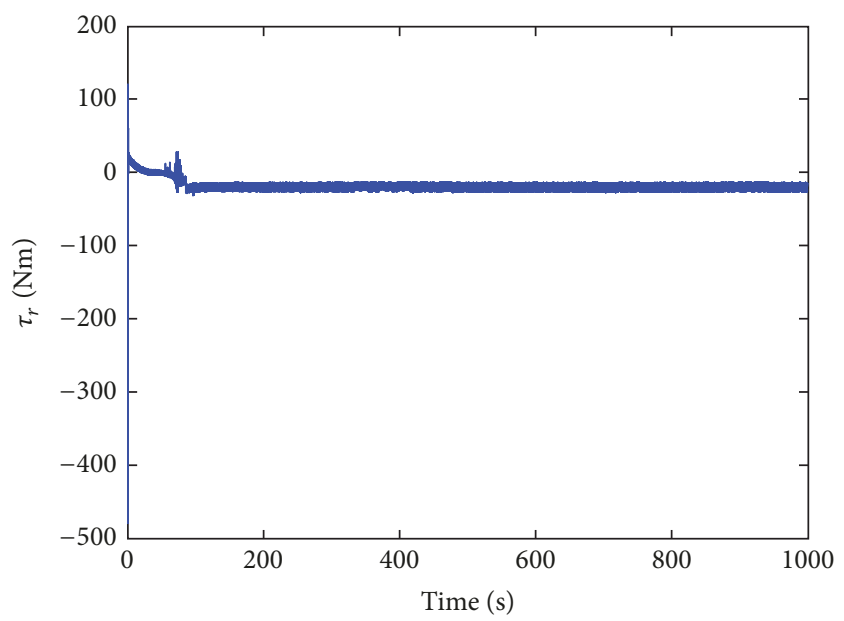

FIGURE 10: Control input $\tau_{r}$ (straight-line). 


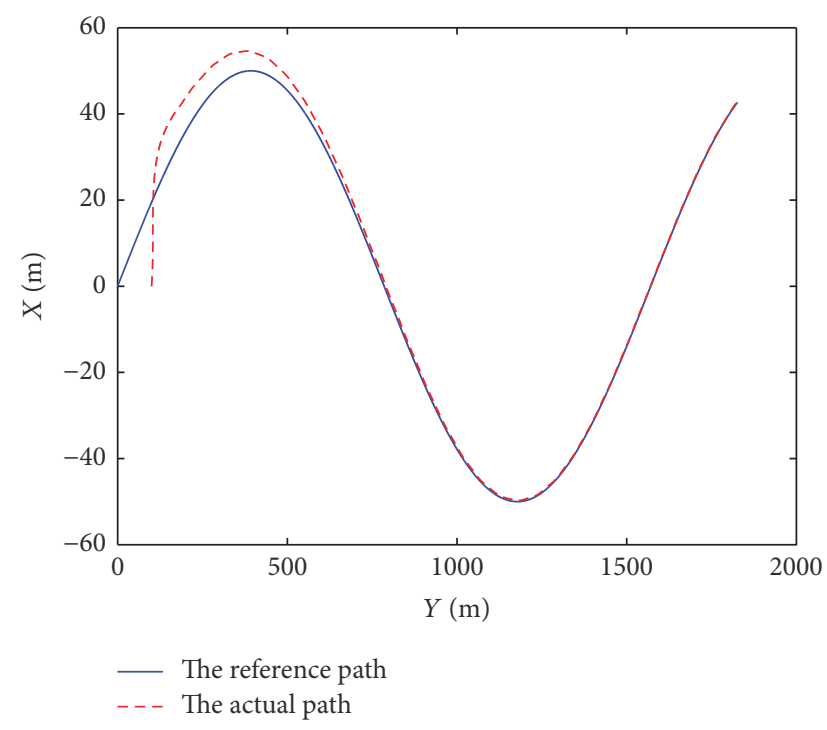

FIGURE 11: Path following performance (curve).

Figure 4 depicts that the USV can accurately follow the desired path very well, but it is impossible that there is no compensation for the sideslip angle. In addition, it can approach the desired path in a relatively short period of time without obvious overshoot. In Figure 5, the actual heading can track the reference heading very well, which shows that the yaw rate controller has better ability to resist external disturbance. Similarly, Figure 6 plots the actual speed $u$. It can reach the target value $1 \mathrm{~m} / \mathrm{s}$ in about 1 second and remain stable, showing that the surge speed controller has good response speed and the ability to resist external disturbance. The curves of $\Delta$ and estimated sideslip angle are shown in Figures 7 and 8 , respectively. It can be seen clearly that their changing rules are in line with the expected theoretical assumptions. Figures 9 and 10 represent the system's control inputs. They take about 1 second to converge to a relatively stable value, and fluctuations always exist to compensate for dynamic uncertainties and external disturbances.

6.2. Curve Path Following Simulation. In order to verify the versatility of the whole system, the curve path following is executed when the control parameters and initial state are not changed. The desired geometrical path is a curve expressed as $p_{d}(\theta)=[50 \sin (\theta / 250), \theta]^{T}$. The simulation results of curve path following are plotted in Figures 11-17.

The result of curve path following is shown in Figure 11. In spite of the external disturbances, USV still has a good following performance without changing any guidance and control parameters. Figures 12 and 13 show the following effects of heading angle and speed $u$. Similar to the straightline path following, heading angle and speed $u$ still have very good control results, which can keep up with the target value in a short time. Hence one can see that yaw rate controller and surge speed controller still maintain good control results, which shows that they have a good adaptability. Figures 14 and 15 plot the values of $\Delta$ and sideslip angle. Control inputs $\tau_{u}$ and $\tau_{r}$ are depicted in Figures 16 and 17, respectively. $\tau_{r}$ of

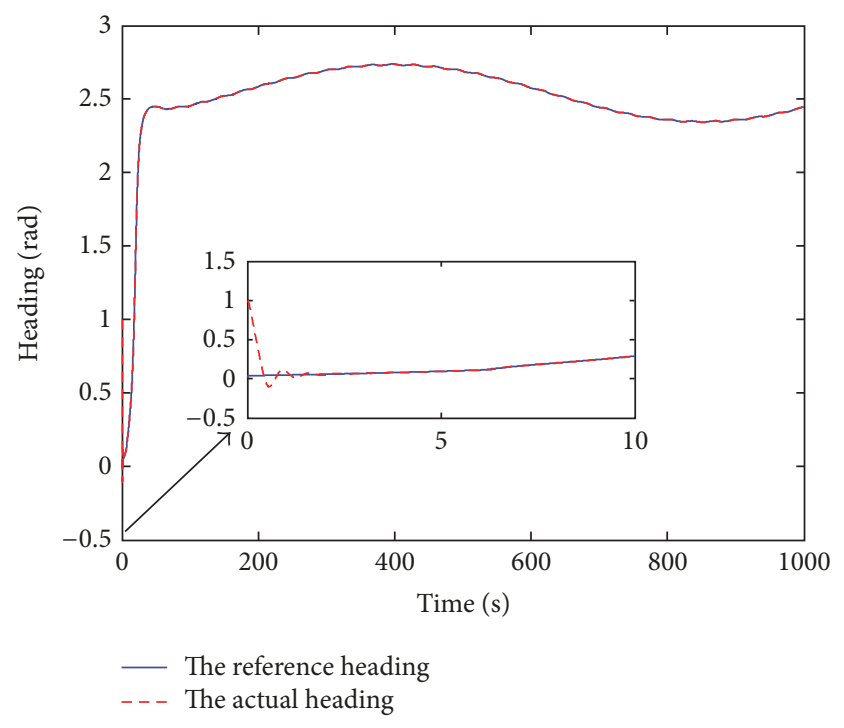

FIGURE 12: Heading control performance (curve).

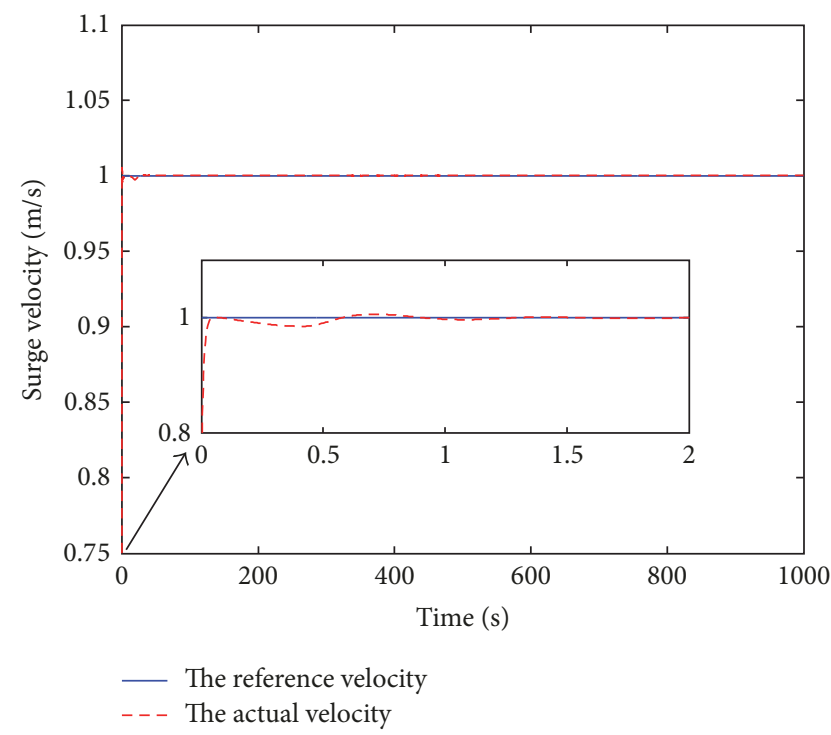

FIGURE 13: Speed control performance (curve).

curve simulation is clearly more obvious than the fluctuation of straight-line simulation. We can see that the curve path following requires a higher requirement for $\tau_{r}$.

Through the above numerical simulations and theoretical analysis, one can see that in the presence of external disturbances USV has a good performance for the same set of guidance and control parameters regardless of whether the desired path is straight-line or curve, which shows the effectiveness and correctness of the whole path following strategy.

\section{Conclusions}

A complete path following strategy is proposed for underactuated USV in this paper. First of all, an optimized LOS algorithm is proposed. Then, in the process of designing the 


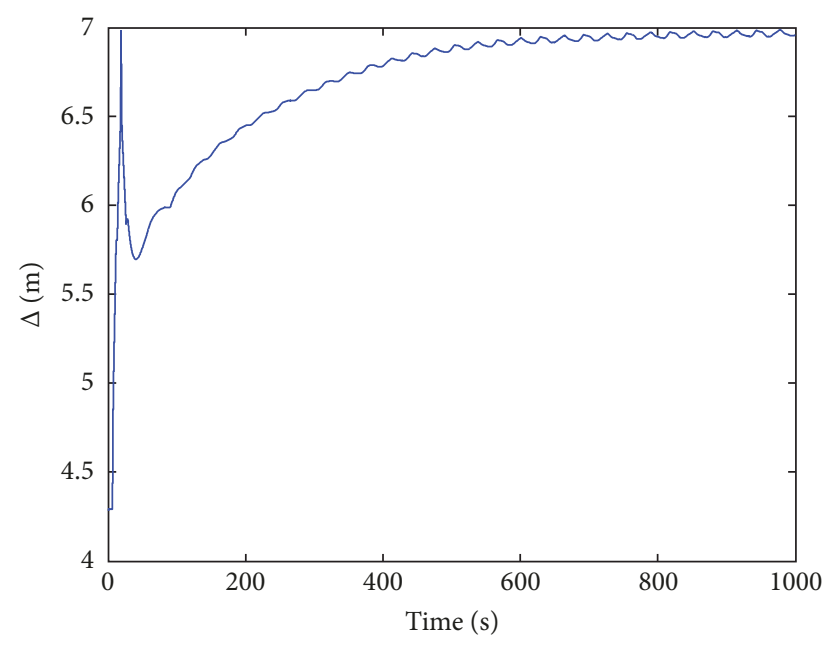

FIgURE 14: The value of $\Delta$ (curve).

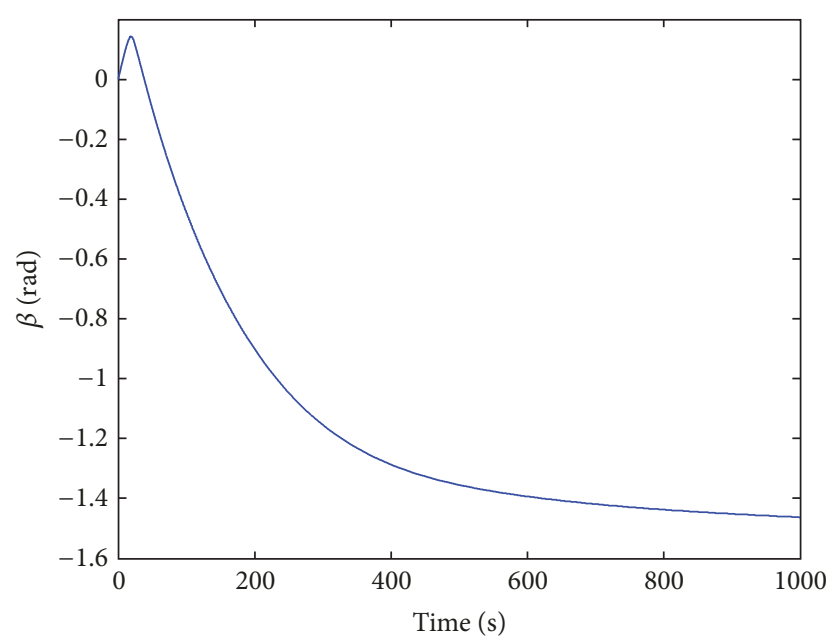

FIGURE 15: The estimated value of sideslip angle (curve).

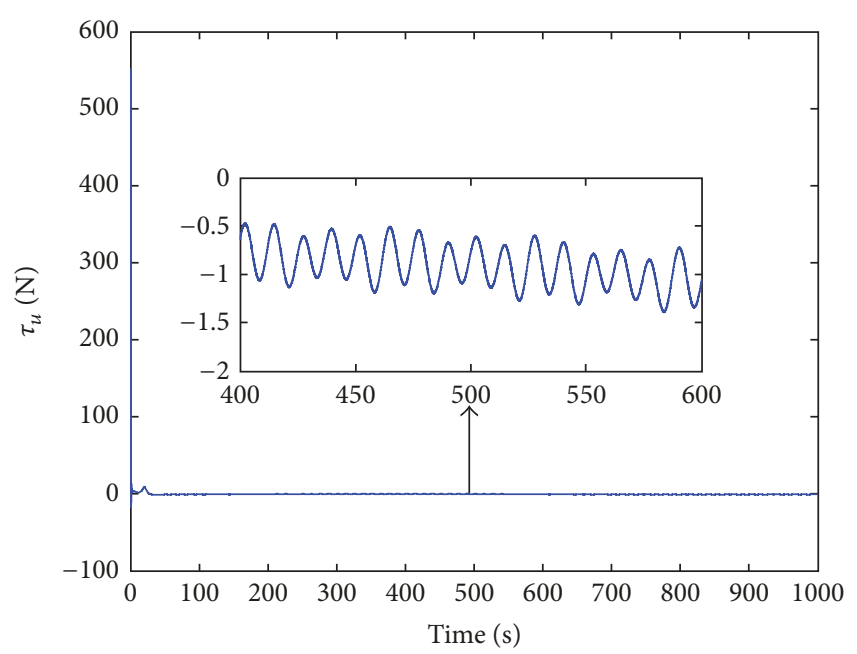

FIGURE 16: Control input $\tau_{u}$ (curve).

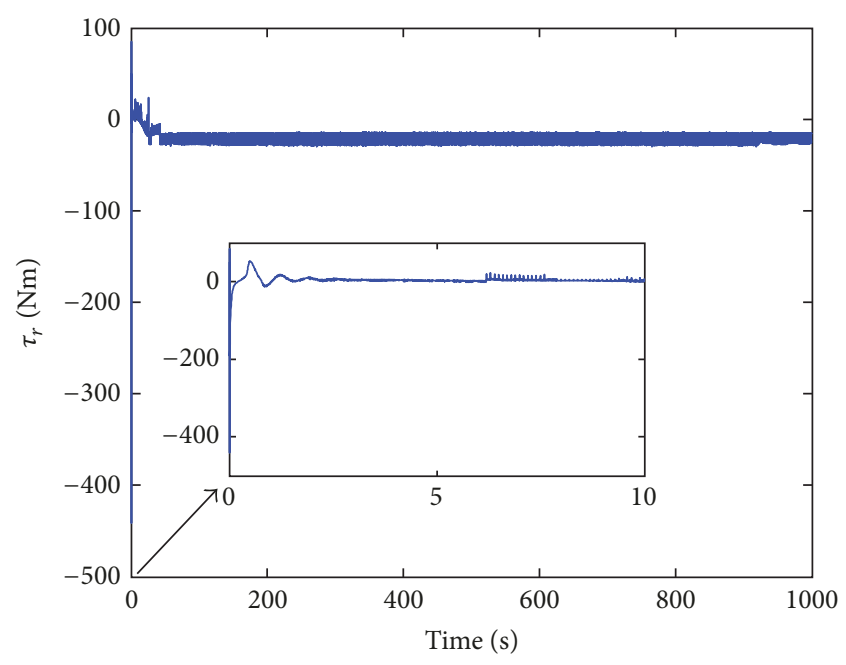

FIGURE 17: Control input $\tau_{r}$ (curve).

controller, the unknown dynamics and the external disturbances are compensated online by neural network minimum parameter learning method. Meanwhile, the "explosion of complexity" problem caused by the derivation of the virtual control law is addressed by introducing the neural shunting model. Aiming at the problem of unknown control gain, Nussbaum function is introduced to guarantee that the control gain is positive. Stability analysis using a Lyapunov function showed that all error signals in the closed-loop system are guaranteed to be uniformly ultimately bounded. Finally, the correctness of the proposed path following strategy for underactuated USV is proved by numerical simulations.

\section{Conflicts of Interest}

The authors declare that they have no conflicts of interest.

\section{Acknowledgments}

This work was partially supported by the Natural Science Foundation of Liaoning Province of China (Grand no. 2015020022), the National Natural Science Foundation of China (Grand no. 51609033), and the Fundamental Research Funds for the Central Universities (Grand nos. 3132014321, 3132016312, and 3132017133).

\section{References}

[1] T. Liu, Z. Dong, H. Du, L. Song, and Y. Mao, "Path Following Control of the Underactuated USV Based On the Improved Line-of-Sight Guidance Algorithm," Polish Maritime Research, vol. 24, no. 1, pp. 3-11, 2017.

[2] Z. Dong, L. Wan, T. Liu, and J. Zeng, "Horizontal-Plane Trajectory-Tracking Control of an Underactuated Unmanned Marine Vehicle in the Presence of Ocean Currents," International Journal of Advanced Robotic Systems, vol. 13, no. 3, 2016.

[3] J. M. Larrazabal and M. S. Peñas, "Intelligent rudder control of an unmanned surface vessel," Expert Systems with Applications, vol. 55, pp. 106-117, 2016. 
[4] X. Yao and G. Yang, "Efficient Multivariable Generalized Predictive Control for Autonomous Underwater Vehicle in Vertical Plane," Mathematical Problems in Engineering, vol. 2016, Article ID 4650380, 2016.

[5] Z. H. Ismail and V. W. E. Putranti, "Second Order Sliding Mode Control Scheme for an Autonomous Underwater Vehicle with Dynamic Region Concept," Mathematical Problems in Engineering, vol. 2015, Article ID 429215, 2015.

[6] Y.-Y. Chen and Y.-P. Tian, "Coordinated path following control of multi-unicycle formation motion around closed curves in a time-invariant flow," Nonlinear Dynamics, vol. 81, no. 1-2, pp. 1005-1016, 2015.

[7] E. W. McGookin, D. J. Murray-Smith, Y. Li, and T. I. Fossen, "Ship steering control system optimisation using genetic algorithms," Control Engineering Practice, vol. 8, no. 4, pp. 429-443, 2000.

[8] T. I. Fossen and K. Y. Pettersen, "On uniform semiglobal exponential stability (USGES) of proportional line-of-sight guidance laws," Automatica, vol. 50, no. 11, pp. 2912-2917, 2014.

[9] M. Breivik and T. I. Fossen, "Path following for marine surface vessels," in Proceedings of the Ocean'04 - MTS/IEEE TechnoOcean'04: Bridges across the Oceans - Conference Proceedings, pp. 2282-2289, jpn, November 2004.

[10] A. Hac and M. D. Simpson, "Estimation of vehicle side slip angle and yaw rate," SAE Technical Papers, 2000.

[11] E. Børhaug, A. Pavlov, and K. Y. Pettersen, "Integral LOS control for path following of underactuated marine surface vessels in the presence of constant ocean currents," in Proceedings of the 47th IEEE Conference on Decision and Control, CDC 2008, pp. 4984-4991, Mexico, December 2008.

[12] W. Caharija, K. Y. Pettersen, M. Bibuli et al., "Integral Line-ofSight Guidance and Control of Underactuated Marine Vehicles: Theory, Simulations, and Experiments," IEEE Transactions on Control Systems Technology, vol. 24, no. 5, pp. 1623-1642, 2016.

[13] K. Y. Pettersen and E. Lefeber, "Way-point tracking control of ships," Proceedings of the IEEE Conference on Decision and Control, vol. 1, pp. 940-945, 2001.

[14] T. I. Fossen, K. Y. Pettersen, and R. Galeazzi, "Line-of-sight path following for dubins paths with adaptive sideslip compensation of drift forces," IEEE Transactions on Control Systems Technology, vol. 23, no. 2, pp. 820-827, 2015.

[15] A. Pavlov, H. Nordahl, and M. Breivik, "MPC-based optimal path following for underactuated vessels," in Proceedings of the 8th International IFAC Conference on Manoeuvring and Control of Marine Craft, MCMC 2009, pp. 340-345, Brazil, September 2009.

[16] A. M. Lekkas and T. I. Fossen, "A time-varying lookahead distance guidance law for path following," in Proceedings of the 9th IFAC Conference on Manoeuvring and Control of Marine Craft, MCMC 2012, pp. 398-403, Italy, September 2012.

[17] A. M. Lekkas and T. I. Fossen, "Integral LOS path following for curved paths based on a monotone cubic hermite spline parametrization," IEEE Transactions on Control Systems Technology, vol. 22, no. 6, pp. 2287-2301, 2014.

[18] H. Wang, P. X. Liu, and P. Shi, “Observer-based fuzzy adaptive output-feedback control of stochastic nonlinear multiple timedelay systems," IEEE Transactions on Cybernetics, 2017.

[19] X. Zhao, P. Shi, X. Zheng, and L. Zhang, "Adaptive tracking control for switched stochastic nonlinear systems with unknown actuator dead-zone," Automatica, vol. 60, article no. 6481, pp. 193-200, 2015.
[20] X. Zhao, H. Yang, and G. Zong, "Adaptive Neural Hierarchical Sliding Mode Control of Nonstrict-Feedback Nonlinear Systems and an Application to Electronic Circuits," IEEE Transactions on Systems, Man, and Cybernetics: Systems, vol. 47, no. 7, pp. 1394-1404, 2017.

[21] K. D. Do and J. Pan, "Global robust adaptive path following of underactuated ships," Automatica, vol. 42, no. 10, pp. 1713-1722, 2006.

[22] J.-H. Li, P.-M. Lee, B.-H. Jun, and Y.-K. Lim, "Point-to-point navigation of underactuated ships," Automatica, vol. 44, no. 12, pp. 3201-3205, 2008.

[23] G. Zhang, X. Zhang, and Y. Zheng, "Adaptive neural pathfollowing control for underactuated ships in fields of marine practice," Ocean Engineering, vol. 104, pp. 558-567, 2015.

[24] G. Zhang and X. Zhang, "A novel DVS guidance principle and robust adaptive path-following control for underactuated ships using low frequency gain-learning," ISA Transactions ${ }^{\circledR}$, vol. 56, pp. 75-85, 2015.

[25] D. Mu, G. Wang, and Y. Fan, "Design of Adaptive Neural Tracking Controller for Pod Propulsion Unmanned Vessel Subject to Unknown Dynamics," Journal of Electrical Engineering Technology, vol. 12, no. 6, pp. 2365-2377, 2017.

[26] H. Wang, X. Liu, and K. Liu, "Adaptive neural data-based compensation control of non-linear systems with dynamic uncertainties and input saturation," IET Control Theory \& Applications, vol. 9, no. 7, pp. 1058-1065, 2015.

[27] D. Mu, G. Wang, Y. Fan, and Y. Zhao, "Modeling and Identification of Podded Propulsion Unmanned Surface Vehicle and Its Course Control Research," Mathematical Problems in Engineering, vol. 2017, Article ID 3209451, 2017.

[28] X. Zhao, H. Yang, W. Xia, and X. Wang, "Adaptive fuzzy hierarchical sliding mode control for a class of MIMO nonlinear time-delay systems with input saturation," IEEE Transactions on Fuzzy Systems, vol. 25, no. 5, pp. 1062-1077, 2017.

[29] H. Wang, W. Liu, J. Qiu, and P. X. Liu, "Adaptive Fuzzy Decentralized Control for A Class of Strong Interconnected Nonlinear Systems with Unmodeled Dynamics," IEEE Transactions on Fuzzy Systems, pp. 1-11.

[30] Z. Zheng and L. Sun, "Path following control for marine surface vessel with uncertainties and input saturation," Neurocomputing, vol. 177, pp. 158-167, 2016.

[31] L. Liu, D. Wang, and Z. Peng, "Path following of marine surface vehicles with dynamical uncertainty and time-varying ocean disturbances," Neurocomputing, vol. 173, pp. 799-808, 2016.

[32] B. Chen, X. Liu, K. Liu, and C. Lin, "Direct adaptive fuzzy control of nonlinear strict-feedback systems," Automatica, vol. 45, no. 6, pp. 1530-1535, 2009.

[33] H. Qu, S. X. Yang, A. R. Willms, and Z. Yi, "Real-time robot path planning based on a modified pulse-coupled neural network model," IEEE Transactions on Neural Networks and Learning Systems, vol. 20, no. 11, pp. 1724-1739, 2009.

[34] S. X. Yang, A. Zhu, G. Yuan, and M. Q.-H. Meng, "A bioinspired neurodynamics-based approach to tracking control of mobile robots," IEEE Transactions on Industrial Electronics, vol. 59, no. 8, pp. 3211-3220, 2012.

[35] R. Yu, Q. Zhu, G. Xia, and Z. Liu, "Sliding mode tracking control of an underactuated surface vessel," IET Control Theory \& Applications, vol. 6, no. 3, pp. 461-466, 2012.

[36] Y. Xudong and J. Jingping, "Adaptive nonlinear design without a priori knowledge of control directions," Institute of Electrical and Electronics Engineers Transactions on Automatic Control, vol. 43, no. 11, pp. 1617-1621, 1998. 
[37] K. S. Narendra and A. M. Annaswamy, Stable adaptive systems, Prentice Hall, Stable adaptive systems, 1989, pp. 579-588.

[38] C. Chen, Z. Liu, Y. Zhang, C. L. P. Chen, and S. Xie, "Saturated Nussbaum Function Based Approach for Robotic Systems with Unknown Actuator Dynamics," IEEE Transactions on Cybernetics, vol. 46, no. 10, pp. 2311-2322, 2016.

[39] Y.-J. Liu and S. Tong, "Barrier Lyapunov functions for Nussbaum gain adaptive control of full state constrained nonlinear systems," Automatica, vol. 76, pp. 143-152, 2017.

[40] R. D. Nussbaum, "Some remarks on a conjecture in parameter adaptive control," Systems \& Control Letters, vol. 3, no. 5, pp. 243-246, 1983.

[41] H. Qi, Z. Zhang, J.-T. Xu, P.-X. Han, and D.-W. Zhang, "Integrated guidance and control of missile based on Nussbaum gain adaptive sliding mode control method," Control and Decision, vol. 32, no. 1, pp. 93-99, 2017.

[42] H.-d. Chen and P. Jiang, "Adaptive iterative feedback control for nonlinear system with unknown control gain," Control Theory \& Applications. Kongzhi Lilun yu Yingyong, vol. 20, no. 5, pp. 691-694, 2003.

[43] J.-L. Du and C. Guo, "Nonlinear adaptive design for coursetracking control of ship without a priori knowledge of control gain," Kongzhi Lilun Yu Yingyong/Control Theory and Applications, vol. 22, no. 2, pp. 315-320, 2005.

[44] S.-L. Dai, C. Wang, and F. Luo, "Identification and learning control of ocean surface ship using neural networks," IEEE Transactions on Industrial Informatics, vol. 8, no. 4, pp. 801-810, 2012.

[45] C.-Z. Pan, X.-Z. Lai, S. X. Yang, and M. Wu, "An efficient neural network approach to tracking control of an autonomous surface vehicle with unknown dynamics," Expert Systems with Applications, vol. 40, no. 5, pp. 1629-1635, 2013. 


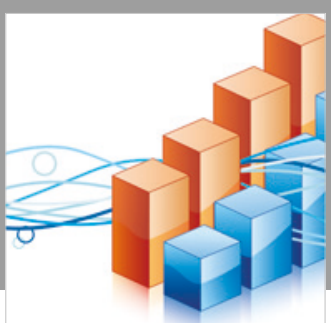

Advances in

Operations Research

\section{-n-m}
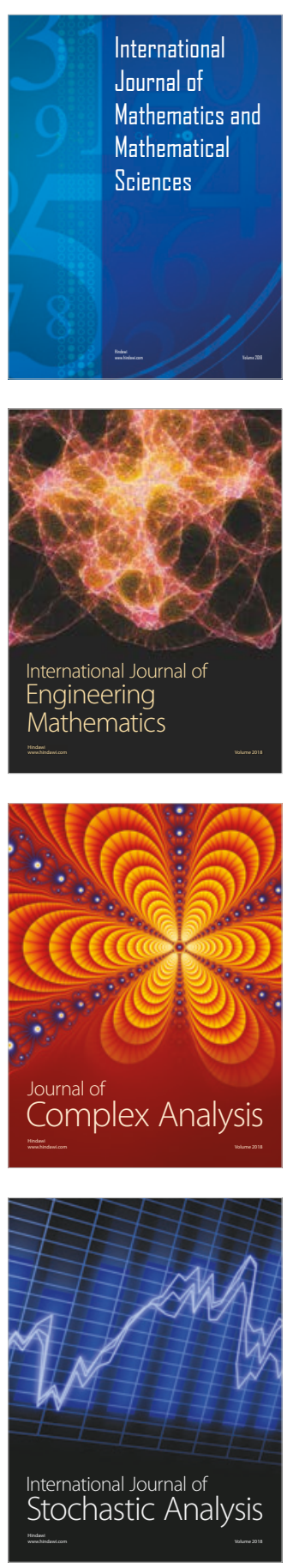
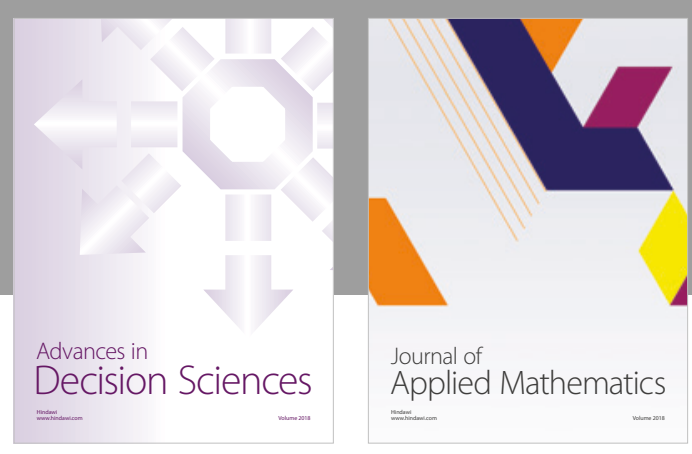

Journal of

Applied Mathematics
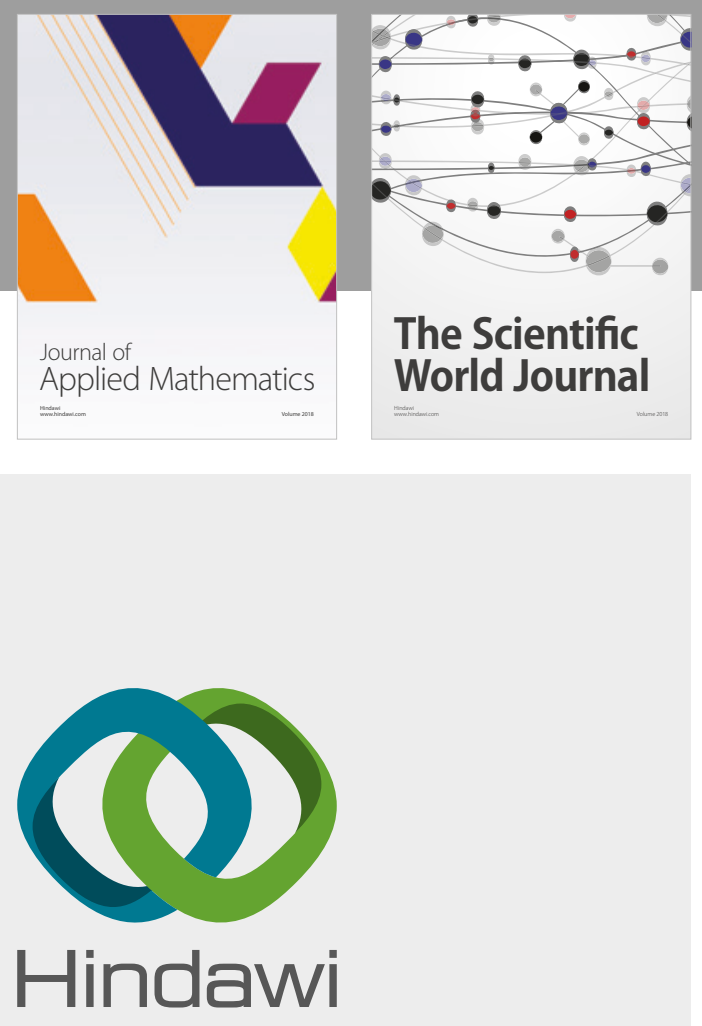

Submit your manuscripts at

www.hindawi.com

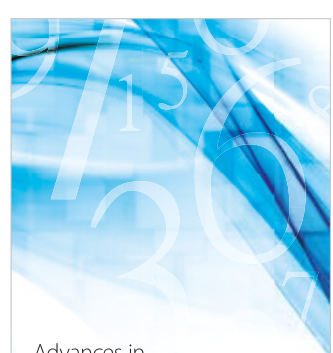

Advances in
Numerical Analysis
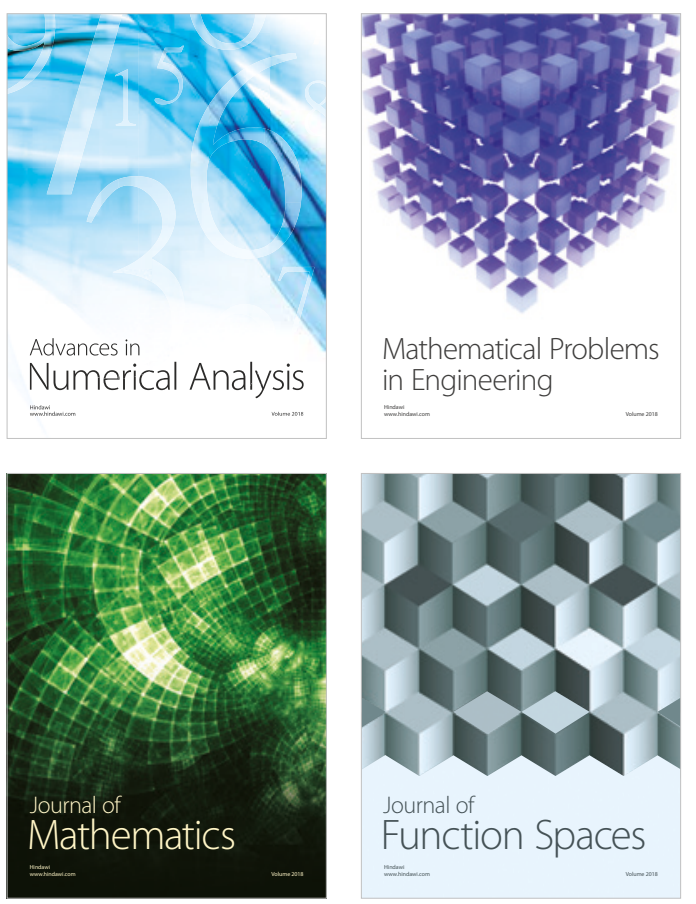

Mathematical Problems in Engineering

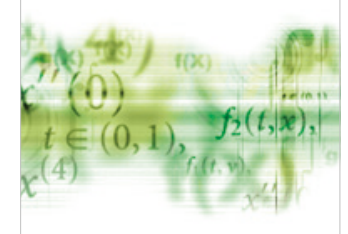

International Journal of

Differential Equations

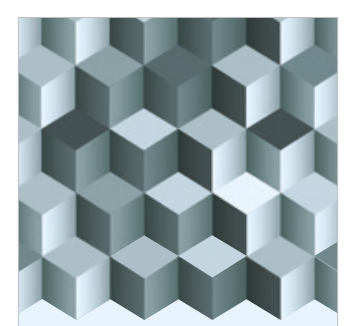

Journal of

Function Spaces

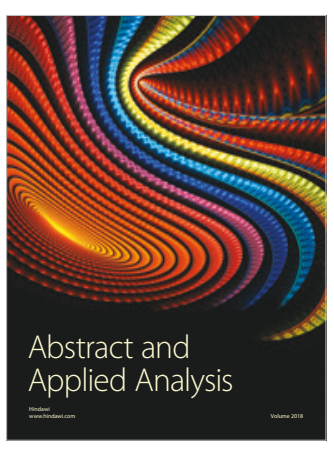

The Scientific

World Journal

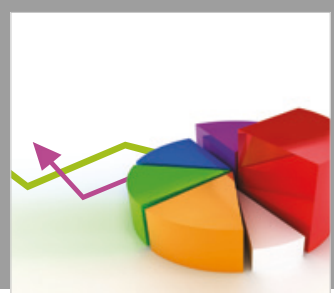

Journal of

Probability and Statistics
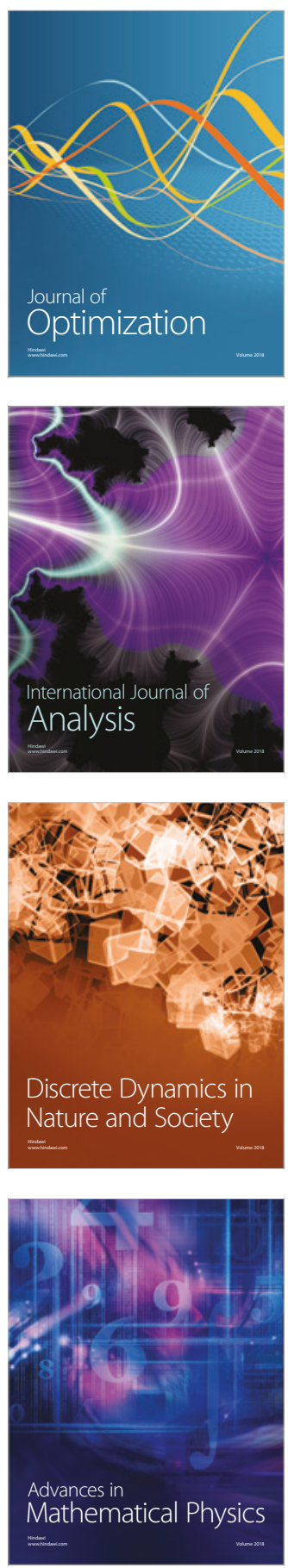